\title{
Estimating the minimum grip force required when grasping objects under impulsive loading conditions
}

\author{
Y. N. TURRELL, F.-X. LI, and A. M. WING \\ University of Birmingham, Birmingham, England
}

\begin{abstract}
As an aid to studying the efficiency of grip force scaling in the context of collisions, we present a simple cost-effective approach to estimating the slip ratio- that is, the minimum grip-to-load-force ratio needed to prevent object slippage. The grip apparatus comprises a sturdy load cell to measure grip force and two linear potentiometers to provide detailed description of finger movements. The slip ratio was estimated by plotting the magnitude of finger movement against the grip-to-load-force ratio at the time of impact. The slip ratio was dependent on the direction of loading, which stresses the importance of estimating slip ratios in a context similar to that of the experiment in which the efficiency of subjects' behavior is to be assessed.
\end{abstract}

To hold an object stable in precision grip, sufficient grip force (GF) normal to the surfaces of the object must be applied through the fingers to overcome load force (LF) tangential to the surfaces that act on the object owing to gravity or inertia. If GF is not sufficient, the object may accidentally slip or even be dropped. At the same time, excess GF must be avoided in order to prevent muscle fatigue and possible damage to the object. If LF increases and the object begins to slip, sensorimotor feedback mechanisms exist that will increase GF in response to the slip and stabilize the object (Johansson \& Westling, 1987). Normally, however, LF increases are anticipated-whether these arise in lifting (Westling \& Johansson, 1984) or moving (Flanagan, Tresilian, \& Wing, 1995)-and GF rises in parallel with the changes in LF. Such anticipation implies that the central nervous system must possess up-todate sensorimotor memories of the manipulated object (e.g., Gordon, Westling, Cole, \& Johansson, 1993) and of the forces acting on it (e.g., Flanagan \& Wing, 1997; Ito, 1994). By examining the adequacy of GF-to-LF scaling, it is possible to estimate the accuracy and functionality of the predictive mechanisms that control the fine adjustment of GF. To determine the adequacy of GF-to-LF scaling, a method is needed for estimating the minimum GFto-LF ratio required to prevent object slippage. This parameter is equivalent to the inverse of the coefficient of friction and has been denoted as the slip ratio.

LF fluctuations can be slow (e.g., lifting) or more rapid (e.g., point-to-point transport) but can be abrupt and, so, particularly threatening to grasp stability under impulsive

The authors especially thank Steve Allen, Dave MacIntyre, and Dave Symons for their skillful assistance in the design and construction of the grip apparatus. We also thank three anonymous reviewers for helpful comments on a previous version of the manuscript. All correspondence should be addressed to F.-X. Li, School of Sport and Exercise Sciences, Perception-Action Laboratory, University of Birmingham, Edgbaston, Birmingham B15 2TT, England (e-mail: f.x.li@bham.ac.uk). conditions (e.g., collisions). When experiencing a collision between a hand-held object and another (as when using a hammer), there may not be sufficient time for sensorydriven adjustment of GF to restore grasp stability. Consequently, under impulsive loading conditions, performance of the action is likely to depend on the capacity to precisely anticipate the nature of the destabilizing forces. Methods for evaluating the accuracy of these predictive mechanisms against the minimum GF-to-LF ratio needed for grasp stability are thus likely to be relevant, both in theoretical studies and in applied behavioral research on motor skill acquisition, in tasks involving collisions - including, for example, sports (tennis, baseball, hockey), music (percussion), and ergometry (hammer). To date, the slip ratio has been estimated only under slow loading. As the value of the slip ratio may be dependent on the loading conditions, it was considered important to develop a paradigm for estimating slip ratios under impulsive loading.

Three techniques have been described for estimating the slip ratio under slow loading conditions. The most widespread approach is to ask subjects to slowly separate the thumb and the index finger until the object drops (Cadoret \& Smith, 1996; Cole, Rotella, \& Harper, 1999; Häger-Ross, Cole, \& Johansson, 1996; Jenmalm \& Johansson, 1997; Johansson \& Westling, 1984, 1987, 1988; Jones \& Hunter, 1992; Westling \& Johansson, 1984; Wing \& Lederman, 1998). An accelerometer placed on the top of the object detects object movement, and the slip point is defined as the first detectable change in the object's position. At this moment in time, GF is measured and is defined as the minimum GF needed to avoid object slippage. In another method (Burstedt, Edin, \& Johansson, 1997; Edin, Westling, \& Johansson, 1992), the slip ratio is determined by instructing subjects to gradually loosen their grip on the object and tilt it slightly until a slip occurs one digit at a time. Cole and Johansson (1993) proposed a slightly different approach, in which subjects were asked to place their index finger on a grip surface and in- 
crease their finger flexion to at least $1 \mathrm{~N}$, while the manipulandum was servo-regulated to a constant position. Subjects then dragged their index fingers proximally while maintaining the finger flexion force until one or more slips occurred. In this fashion, static slip ratio was precisely determined for each individual digit by detecting vibrations in the positional signals induced by finger slip. These methods use acceleration to identify slip onset from vibration signals. They have proven to be very useful approaches, well suited to estimate slip ratios in tasks for which vibration signals can be attributed to an object's change of position.

When an object that is subject to a collision is held, large and sudden LF increases are applied to the held object, giving rise to significant stress waves (i.e., highfrequency vibrations) that propagate throughout the system (object, hand, and arm). The use of acceleration signals to monitor object slippage is thus limited, since the signals arising from the object's change in position are drowned within the vibrations arising from the impact. To investigate the efficiency of behavior in collisions, it was necessary to design a device that could be used to estimate the minimum GF required to prevent slips in manipulative tasks involving large and impulsive LFs. A second concern was to limit the cost of the approach: The apparatus described in the following can be constructed for a few hundred dollars. In the present report, we describe the design and function of the prototype of a grip apparatus that enables (1) detection and measurement of the magnitude of finger slips and (2) recording of GF fluctuations during manipulative tasks involving collisions of various magnitudes. With such a device, it was then possible to estimate the slip ratio. After controlling for symmetrical loading of the fingers, the usefulness of the grip apparatus is demonstrated by estimating slip ratios under different loading conditions.

\section{APPARATUS}

The grip apparatus comprised two parallel woodsurfaced plates ${ }^{1}$ (width, $4.5 \mathrm{~cm}$; length, $12.2 \mathrm{~cm}$ ), between which was mounted a load cell (Novatech Model F245). This one-axis load cell measured the GF produced by the digits normal to the surfaces of the two plates. Two linear potentiometers (Alps STRSA0N12S; sensitivity of $0.1 \mathrm{~mm}$ )—including shaft, slider, and ring-were mounted parallel to either side of the gripped surfaces (Figure 1). Individual copper rings (padded with wax moulds) were fitted around the distal phalanges of the thumb and middle finger and coupled the tip of the digit to each potentiometer's slider. As the finger-ring-slider complex moved up and down the shaft of each potentiometer, voltage varied, giving a precise measurement of change in finger position. The total weight of the grip apparatus was $545 \mathrm{~g}$.

\section{ASSESSING SYSTEM PERFORMANCE}

A VICON optoelectronic motion analysis system was used to obtain an independent measurement of position signals (three cameras, $120-\mathrm{Hz}$ sampling rate). The accuracy of the cameras was $0.1 \%$ of the volume (i.e., approximately $0.1 \mathrm{~mm}^{3}$ ). Five reflective markers were placed: one on the nail and one on the proximal interphalangeal joint of the thumb, one on the potentiometer slider, and one on each extremity of the potentiometer (Figure 1B). Three-dimensional (3-D) displacements were reconstructed for every marker at the end of each trial. For all the markers, the variation along the $y$-axis was negligible. Positional and tangential velocity-time functions along the $x$-axis were derived and used to determine the start and end of movement (defined as the first moment in time when the velocity exceeded $10 \mathrm{~mm} / \mathrm{sec}$ ). Similar results were found when tracking either marker on the thumb and that placed on the slider, indicating that the different manipulative tasks did not affect the relative positions of the thumb and the ring. In the following sections, the results obtained with the linear potentiometers are compared with those recorded by the cameras tracking the marker on the thumbnail only.

\section{Testing Repeatability}

A series of 40 trials were recorded, using a single subject (26-year-old female) to test the reliability of the measurements obtained with the grip apparatus. The subject used three fingers and the thumb of the right hand to hold the grip apparatus, with the $x$-axis aligned vertically (as shown in Figure 1B). Her task was to reduce GF until the object slipped under the effect of gravity. She was instructed to allow slip magnitude to vary between trials. To simulate separate experiments, the apparatus was placed down and picked up in between each trial, and markers were replaced every 10 trials.

A comparison of the results obtained for the camera and the potentiometer system is presented in Figure 2. The least squares best-fit regression line was given by $y=+1.044 x$. Note that the regression line crossed the $(0,0)$ point of origin, which indicates the absence of bias. Furthermore, the slope (1.044) was very close to the ideal gain of 1. Finally, the measurements were reliable $\left(r^{2}=\right.$ $.990)$, with all the points falling within a confidence interval of $2 \mathrm{~mm}$.

\section{Characterizing a Finger Slip}

The linear potentiometers mounted on the grip apparatus had a sensitivity of $0.1 \mathrm{~mm}$, and thus the slightest finger movement was recorded. However, from the signal it was not clear whether the finger had actually slipped or only rolled under the effect of the impact. When applying a small and constant force at the top of the held object, it was observed that the skin on the fingerpads first rolled, before a sudden and visible slip occurred (Figure 3). When objects are manipulated, only the occurrence of a slip is threatening to grasp stability. Thus, from a behavioral point of view, it was considered important to develop a method for differentiating roll (rotation) and slip (translation) components.

One subject (26-year-old female) fitted the thumb and the middle finger within the rings of the grip apparatus, 


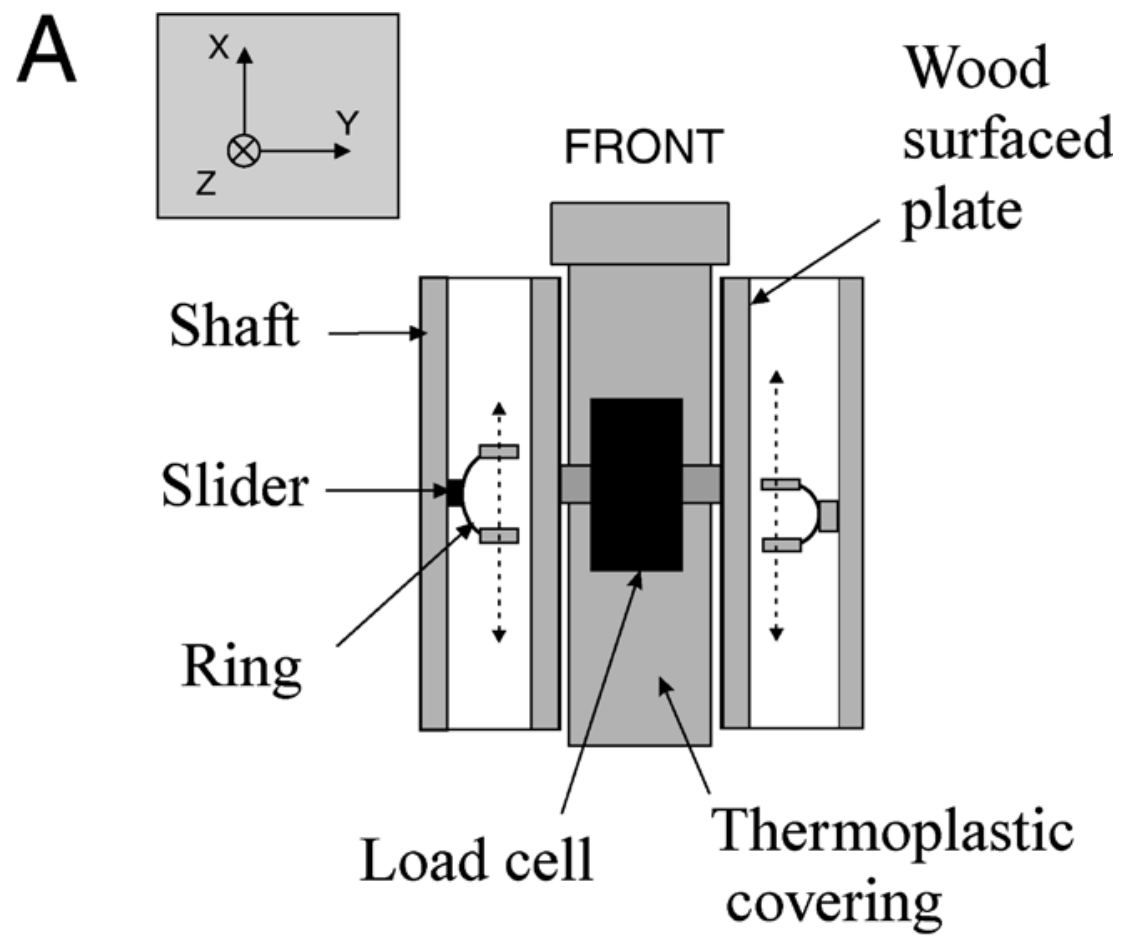

B

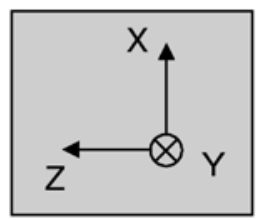

FRONT

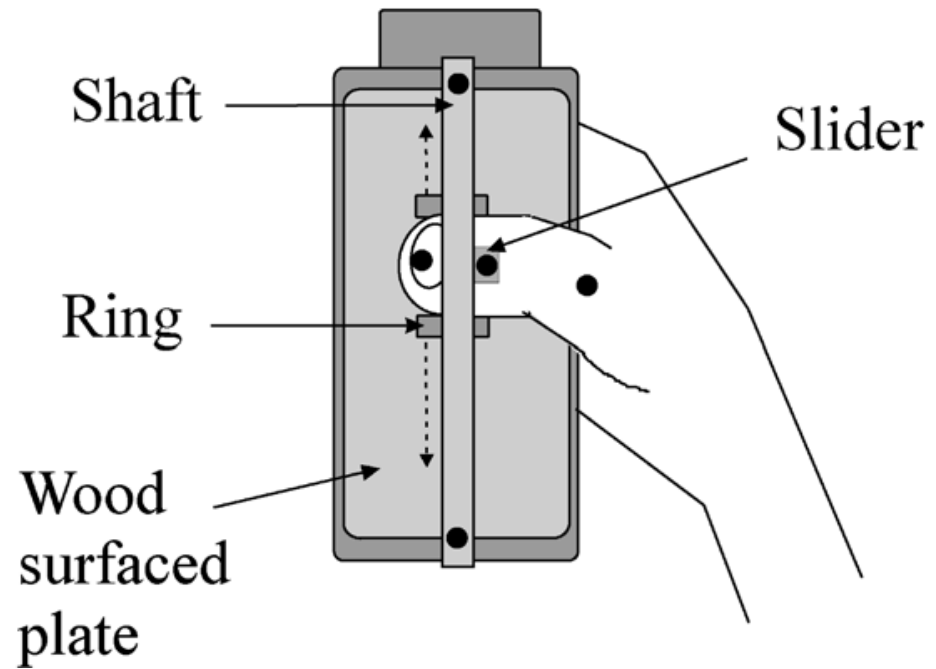

Figure 1. (A) Schematic representation of the grip apparatus (top view), depicting the load cell and the two linear potentiometers (shaft, slider, and ring) that allowed the measurement of the magnitude of movement between fingertip and object surface. The thumb and the middle finger of the right hand were fitted in the left and right rings, respectively. (B) Side view of the grip apparatus, which shows the hand position adopted in the vertical collisions. Full circles depict the positions of the reflective markers used with the VICON system. Dashed arrows in panels A and B describe the displacements of the rings when the fingers move. 


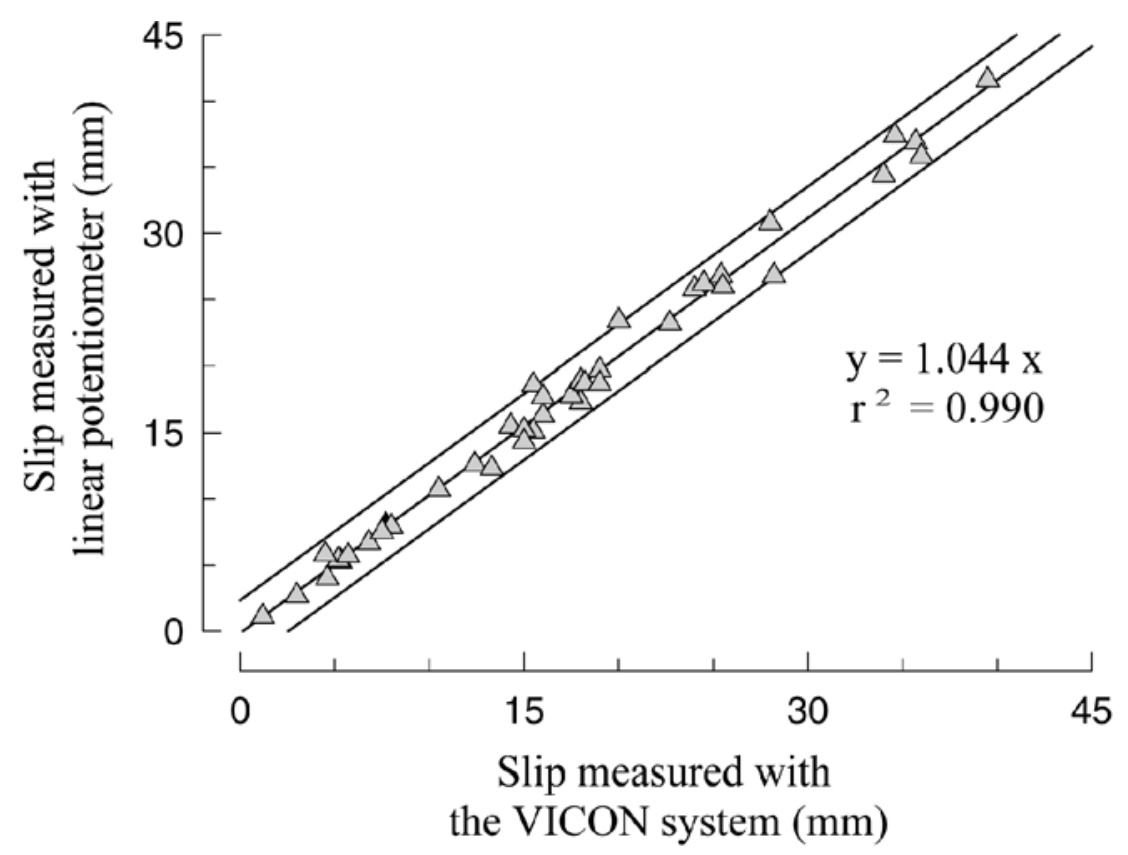

Figure 2. Data from 40 trials with a single subject, showing reliability of measurement. For each trial, slip measurements obtained with the VICON system $(x$-axis) are plotted against those obtained with the grip-apparatus $\left(y\right.$-axis). $r^{2}$ is an indicator of the reliability; the slope of the regression line is an indicator of the linearity of the measurements.

which was set on a table so that the $x$-axis of the apparatus was horizontal. Maintaining her fingers within the rings, she forced her thumb in each direction so that it rolled without slip. A close look at the recorded signals revealed that finger rolls were relatively slow movements limited to a maximum amplitude of $3 \mathrm{~mm}$. In a second task, the grip apparatus was oriented with the $x$-axis vertical (as in Figure 1B). The subject was asked to relax her grip until the held object slipped within her grasp. This procedure was repeated several times, and the results showed that finger slips were characterized by sharp displacement of finger position and could range from 1 to $60 \mathrm{~mm}$. In consideration of these results and the confidence interval determined in the previous section, a slip was defined as a finger movement equal to or greater than $2 \mathrm{~mm}$.

\section{Controlling for Equal Force Distribution}

The mass of the grip apparatus required a multidigit grasp to prevent fatigue. Multidigit grasp raises the problem that individual digits may slip differentially. Flanagan, Burstedt, and Johansson (1999) have recently shown that the distribution of loads across the digits in a multidigit grasp is quite complex. It is therefore possible, in the present case, that the load forces were unequally distributed across the individual fingers used to oppose the thumb. Thus, slips were defined by assessing thumb movements only, on the assumption that each side was doing half of the work in resisting the load and friction. ${ }^{2}$ To verify this latter point, a short experiment was con- ducted. If both digits involved in holding an object in precision grip are equally loaded, the friction at both contact surfaces is equal, and thus, slips should occur in synchrony. Conversely, if there is an unequal force distribution, the friction will be smaller at the relatively unloaded digit, and consequently this digit will not be able to sustain as much force before slipping. Consequently, if an object is held with unequal force distribution, the unloaded digit should slip before the loaded one.

Two series of trials were collected. In the first series, the $x$-axis of the grip apparatus was oriented vertically, which should have led to a symmetrical loading of the fingers and the thumb. In the second series, subjects inclined the grip apparatus by $45^{\circ}$ toward the fingers in such a way that the thumb was relatively unloaded. Forty trials were recorded in 2 subjects (a 52-year-old male and a 26-year-old female). The task was first to hold the grip apparatus between the middle finger and the thumb (two-digit grasp) and then to slowly relax the grip on the object until a slip occurred. Twenty trials were recorded before the subjects were asked to use a multidigit grasp (i.e., three fingers and thumb) for the remaining 20 trials.

The results were similar for both subjects. In the case in which the thumb was relatively unloaded - that is, the condition in which the grip apparatus was inclined-the thumb slipped systematically before the middle finger. The delay between the start of digit movement was, on the average, $67 \mathrm{msec}(S D=55 \mathrm{msec})$ and $69 \mathrm{msec}(S D=$ $53 \mathrm{msec}$ ) for the two-digit and the multidigit grasp configurations, respectively. Time to peak velocity was sig- 


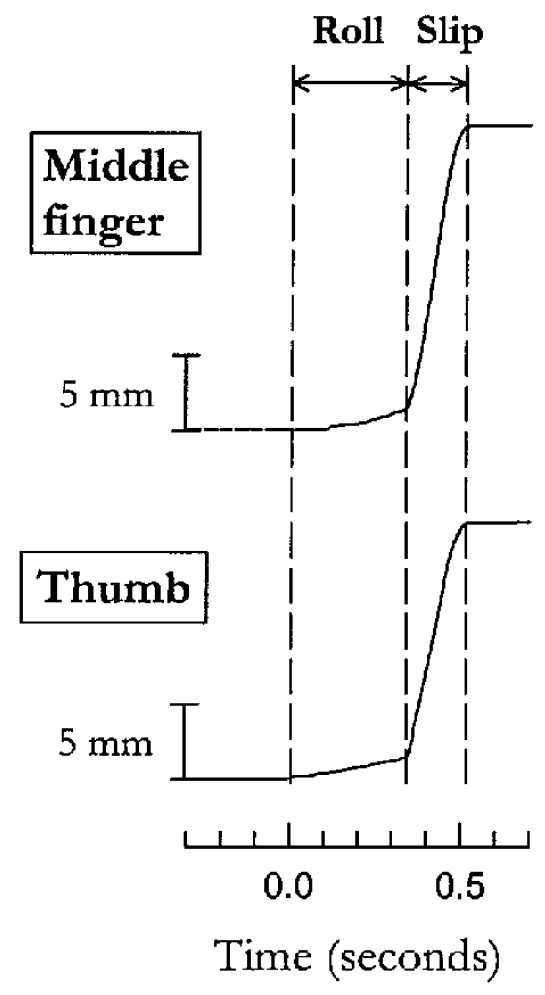

Figure 3. Displacement curves for the middle finger (top) and the thumb (bottom), measured with the linear potentiometers in a task in which the grip apparatus was held vertically and slowly released. Note that the fingers first started to roll. When the tangential forces applied through the fingers were not sufficient to overcome the force of gravity, the fingers slipped. Since the thumb and the finger were equally loaded, the slips were synchronized.

nificantly longer for the thumb than for the finger in both the two-digit ( $144 \mathrm{msec}[S D=47 \mathrm{msec}]$ vs. $69 \mathrm{msec}$ $[S D=14 \mathrm{msec}])$ and the multidigit $(116 \mathrm{msec}[S D=$ $44 \mathrm{msec}]$ vs. $72 \mathrm{msec}[S D=31 \mathrm{msec}]$ ) grasps. When the grip apparatus was oriented vertically, slip onsets occurred in synchrony at both digits. The thumb slipped before the middle finger in $55 \%$ of the trials, and the delay between slip onsets of the two digits was $9 \mathrm{msec}(S D=$ $4 \mathrm{msec})$ and $10 \mathrm{msec}(S D=6 \mathrm{msec})$ for the two-digit and multidigit grasp configurations, respectively. Times to peak velocity were similar for both digits $(62 \mathrm{msec}[S D=$ $36 \mathrm{msec}$ ] vs. $63 \mathrm{msec}[S D=19 \mathrm{msec}]$ and $68 \mathrm{msec}[S D=$ $43 \mathrm{msec}$ vs. $65 \mathrm{msec}$ [ $S D=42 \mathrm{msec}$ ] for the two-digit and multidigit grasps, respectively).

From these results, it is concluded that when the grip apparatus is held aligned with the force of gravity, there is close to equal force distribution between the thumb and the fingers. This is true both when the grip apparatus is held between the middle finger and the thumb and when it is held between three fingers and the thumb, which implies that, in both configurations, the thumb sustains approximately half of the load. With a reliable technique to measure finger slip in the context of collisions and to monitor equal force distribution across the fingers and the thumb, the minimum GF needed to avoid object slippage, for a given type of collision, can be accurately and reliably determined. In the following section, we present a detailed example of how this method can be used to assess whether the slip ratio remains constant when the loading characteristics of a collision are modified.

\section{DATA-BASED EXAMPLE}

In a previous report, we have shown that people are better at scaling anticipatory adjustments of GF to impulsive LF increases in collisions when they produce the collision than when they receive it (Turrell, Li, \& Wing, 1999). We proposed that the better scaling in the collisions that are produced reflects more direct activation of an internal forward model, allowing a more accurate estimate of the consequences of the collisions. In that study, we assumed that the objective slip ratio would be the same in collisions that are produced and those that are received. The following experiment was designed to test this assumption. A second contrast involving hand orientation was introduced. Depending on whether the grip apparatus is aligned horizontally or vertically, shear forces at the digit tips arising from gravitational LF are aligned either at right angles or in line with the direction of the shear forces arising from collision LF. Jones and Hunter (1992) suggested that the friction between the skin and an object being grasped changes as a function of the direction of the force that the object applies to the skin, possibly owing to the anisotropic nature of glabrous skin. We were therefore interested to know whether slip ratios estimated in collisions changed as a function of the orientation of the grasp apparatus.

\section{Method}

Subjects and Task. Four right-handed subjects participated in this experiment ( 2 women and 2 men, 26, 50, 26, and 52 years of age, respectively). All the subjects gave their informed consent before participating in the study. For each type of collision, the subjects performed 30 trials.

On each trial, the subjects held the grip apparatus in their right hands between three fingers on one side and the thumb on the other. They were instructed to vary the level of GF used so that slips would occur as a consequence of the increase in impulsive LF. At the beginning of the session, the subjects washed their hands with soap and water and dried them with paper towels. Between each condition, a 2-5 min break was taken, and hands were wiped with a clean piece of paper towel before resuming the session. This procedure was adopted to limit the effects of individual differences in rate of sweating.

Experimental conditions. The experimental session involved collisions between the grip apparatus and a single-axis load cell (Novatech Model F241) that measured force of impact. The characteristics of the collision were modified by changing the orientation of the impact (vertical vs. horizontal) and the object that was in motion (grip apparatus vs. load cell). For the vertical receiving condition, the subjects held the grip apparatus as illustrated in Figure 1B. With their left hands, they used the load cell to hit the top end of the grip apparatus. For the horizontal collisions, the grip apparatus was held with the $x$ axis oriented horizontally, and the load cell was attached to the bottom end of a pendulum. In the producing task, the subjects used the 
grip apparatus to hit the pendulum to make it swing up to various angles. In the receiving task, the subjects used their left hands to release the pendulum from different angles (for more detail, see Turrell et al., 1999). For the three types of collisions, the durations of the contact were similar and lasted between 15 and $18 \mathrm{msec}$.

Data analysis. LF, pendulum swing, GF, and slip measurements of the thumb and the middle finger were sampled at $1000 \mathrm{~Hz}$. Figure 4 presents typical traces observed in the three types of collisions-vertical receiving (A), horizontal receiving (B), and horizontal producing (C). The LF was calculated as the vectorial sum of the peak force of impact and the mass of the grip apparatus. GF was measured $10 \mathrm{msec}$ before impact in order to avoid the vibration induced by the collision. Finger displacement was measured as the difference between slider positions $30 \mathrm{msec}$ before and $30 \mathrm{msec}$ after impact. The arrows on the graph pick out these particular points. Trials in which the delay between movement initiations of the middle finger and of the thumb was greater than $15 \mathrm{msec}$ were discarded. Fewer than $5 \%$ of the trials were discarded following this criterion.

For each subject and type of collision, the GF-to-LF ratio was calculated, to construct a graph with slip magnitude plotted against force ratio (Figure 5). Trials for which the thumb slipped by $2-4 \mathrm{~mm}$ were then selected, and the GF-to-LF ratios were averaged to obtain a mean slip ratio. Paired $t$ tests were used to assess whether the slip ratios were similar between subjects and types of conditions. The significance level was set at $\alpha=.05$.

\section{Results and Discussion}

The slip ratios calculated for each subject and type of collision are presented in Table 1 . The main effect of subject was significant: Slip ratios were significantly higher for the older than for the younger subjects ( 0.49 and 0.40 vs. 0.15 and 0.16$)$. Older people tend to have drier skin, and thus, the coefficient of friction between fingertips and object surface can be expected to be smaller in older than in younger subjects (Cole, 1991). The main effect of type of collision also was significant. Slip ratios were similar for the horizontal producing and the receiving tasks, which suggests that hand movement does not affect the frictional conditions between fingertips and object surfaces. In contrast, slip ratios were significantly smaller for horizontal collisions than for vertical ones. Because of the use of linear potentiometers, only slips parallel to the loading direction could be recorded. For horizontal collisions, vertical slips could not be recorded, which may have led to systematic underestimation of the slip ratio. This seems unlikely, however, since the force of impact was considerably greater than the force of gravity. If the impact was strong enough to induce a slip, the object should first have slipped horizontally before falling vertically. A more likely explanation is that the viscoelastic and compliant properties of the skin at the fingertips varied with hand posture.

\section{GENERAL DISCUSSION}

To prevent an object held in precision grip from slipping during a collision, GF needs to be scaled to the LF from impact. It is interesting to evaluate how efficient subjects are in such a situation. This requires the estimation of the slip ratio - that is, the minimum GF-to-LF ratio that just prevents slip. We have presented a simple but cost-effective device that allows the recording of GF fluctuations and changes in finger position relative to the ap-

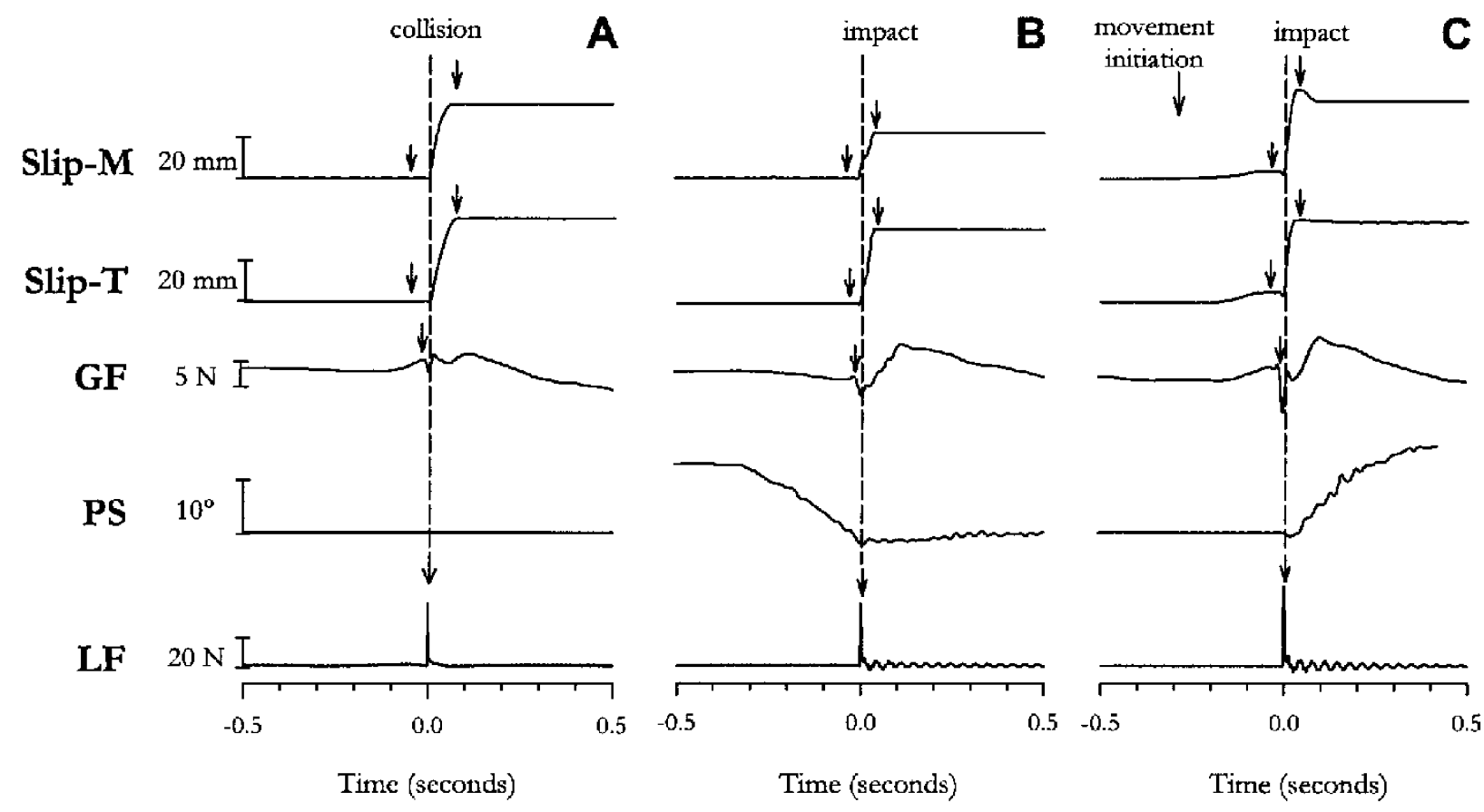

Figure 4. Typical trials observed for collisions in the vertical receiving (A), horizontal receiving (B), and horizontal producing (C) conditions. From top to bottom: slips occurring at the middle finger (Slip-M) and thumb (Slip-T) (as measured by the linear potentiometers), grip force (GF), pendulum swing (PS), and load force (LF). Arrows pick out the points used to calculate the slip ratio. 


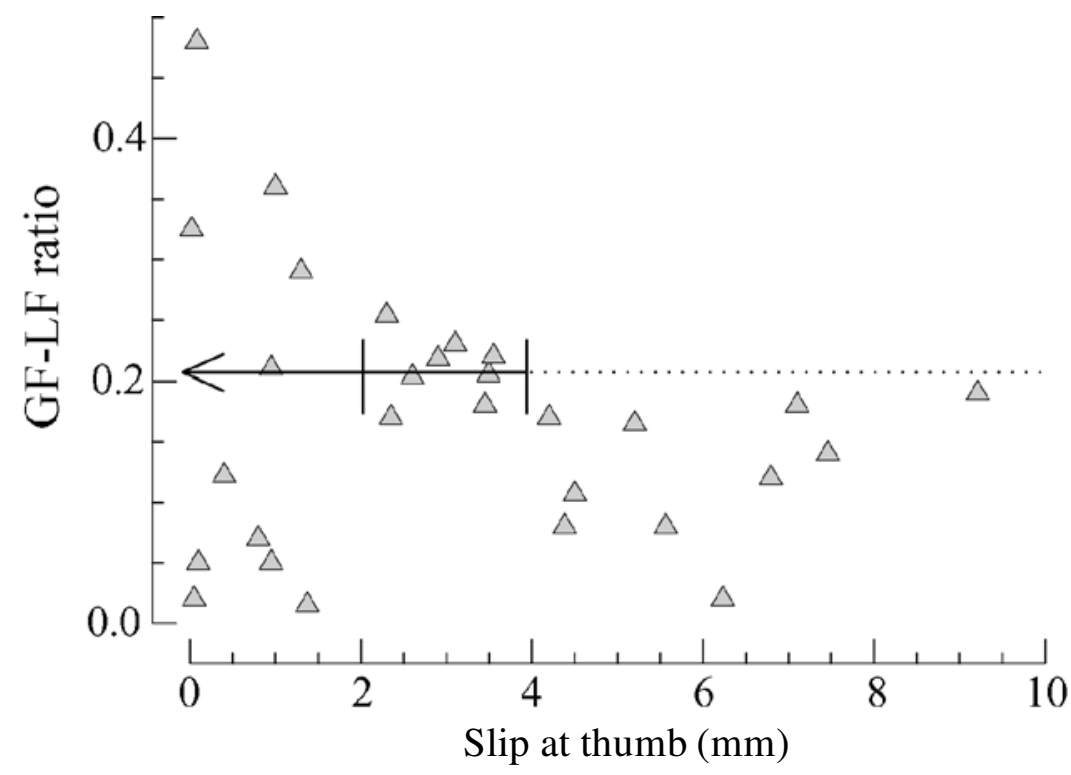

Figure 5. Results are presented for the 26-year-old male subject when vertical collisions were received. For each trial, the magnitude of thumb slip was plotted against the grip-to-load-force ratio (GF-LF ratio). The trials for which the thumb slipped by 2-4 mm were then selected, and the GF-LF ratios were averaged to obtain the mean slip ratio. This procedure was followed for each subject and for each type of collision.

paratus during trials in which collisions of appreciable magnitude are experienced. Using this device, we described a simple paradigm for estimating the slip ratio under various loading conditions. Our results suggest that the slip ratio is dependent on, at least, the direction of the loading. Other preliminary results obtained in our laboratory (unpublished results) suggest further that the slip ratio may also depend on the rate of loading. Further work would be beneficial for a better understanding of the factors influencing the slip ratio. Nevertheless, the results presented here stress the importance of estimating slip ratios under the same loading conditions as that of the experiment in which the efficiency of subjects' behavior is assessed.

The load cell fitted within the apparatus allowed reliable measurement of GF levels of up to $80 \mathrm{~N}$. This level of force is rarely reached in a one-hand grasp-the maximal precision GF produced by male subjects being, on average, $85 \mathrm{~N}$ (Kumar \& Simmonds, 1994). The grip ap-

Table 1

Means and Standard Deviations for the Slip Ratios for the 4 Subjects

\begin{tabular}{|c|c|c|c|c|c|c|c|}
\hline \multirow[b]{2}{*}{ Gender } & \multirow[b]{2}{*}{ Age } & \multicolumn{2}{|c|}{$\begin{array}{l}\text { Receiving } \\
\text { Vertical }^{*}\end{array}$} & \multicolumn{2}{|c|}{$\begin{array}{l}\text { Receiving } \\
\text { Horizontal } \dagger\end{array}$} & \multicolumn{2}{|c|}{$\begin{array}{c}\text { Producing } \\
\text { Horizontal } \dagger\end{array}$} \\
\hline & & $M$ & $S D$ & $M$ & $S D$ & $M$ & $S D$ \\
\hline M & 52 & 0.84 & 0.04 & 0.36 & 0.06 & 0.28 & 0.02 \\
\hline $\mathrm{F}$ & 50 & 0.94 & 0.19 & 0.12 & 0.01 & 0.23 & 0.02 \\
\hline M & 26 & 0.20 & 0.03 & 0.12 & 0.01 & 0.12 & 0.01 \\
\hline $\mathrm{F}$ & 26 & 0.25 & 0.06 & 0.11 & 0.02 & 0.11 & 0.01 \\
\hline
\end{tabular}

$* \mathrm{LF}=$ weight + force of impact. $\quad{ }^{\dagger} \mathrm{LF}=\sqrt{\text { weight }^{2}+\text { force of impact }}{ }^{2}$. paratus can therefore be used to study collisions of magnitudes covering the spectrum of forces suitable for human subjects. The use of this device in collision experiments thus enables the study of the efficiency of GF scaling under a wide range of loading conditions.

The apparatus allowed recording of slip movements to an accuracy of a couple of millimeters. This was as precise and reliable as that obtained with the 3 -D motion analysis system, but not accurate enough to detect either microslips (see Johansson \& Westling, 1988) or the precise moment of slip initiation. In slow loading conditions, moment-to-moment adjustments of GF are possible, and consequently, it is important to detect the precise moment of slip initiation. In impulsive loading, however, the LF pulse is applied for only a couple of tens of milliseconds, thus preventing an on-line adjustment of GF. Therefore, in the context of collisions, an accurate estimation of the slip ratio requires the detection of the occurrence of slips, but not of the precise moment of slip initiation.

In the present study, the subjects used a precision grip in which the thumb, on one side of the object, was opposed by three digits. It was not possible to measure normal and tangential forces produced at individual digits, and it is likely that the fingers were unequally loaded (see Flanagan et al., 1999). However, whatever the force combination, the thumb must have compensated for the sum of the forces applied through the fingers in order to maintain the grip apparatus parallel to the vertical or the horizontal axis. Consequently, slips were defined by monitoring movements of the thumb only. To obtain a reliable estimation of the slip ratio, it was also necessary to en- 
sure that the thumb on one side of the apparatus and the fingers on the other were equally loaded. With the present design, this was controlled by verifying that the initiations of slips at the middle finger and the thumb were synchronized. This was the case in the large majority of trials (over 95\%). To further improve the accuracy and reliability of the approach, it would be beneficial to provide for the possibility of recording normal and tangential forces (as well as torques) at individual digits. Recently, Flanagan et al. (1999) demonstrated, under slow loading conditions, that six-axis force transducers enabled the estimation of both linear and rotational slip ratios without needing to sense slip directly. However, such force transducers are expensive and not sufficiently robust to withstand impulsive loading in collisions, so the present approach appears more practicable at present.

\section{REFERENCES}

Bowden, F. P., \& TABor, D. (1982). Friction. An introduction to tribology. Malabar, FL: R. E. Krieger.

Burstedt, M. K., Edin, B. B., \& Johansson, R. S. (1997). Coordination of fingertip forces during human manipulation can emerge from independent neural network controlling each engaged digit. Experimental Brain Research, 117, 67-79.

Cadoret, G., \& Smith, A. M. (1996). Friction, not texture, dictates grip forces used during object manipulation. Journal of Neurophysiology, 75, 1963-1969.

CoLE, K. J. (1991). Grasp force control in older adults. Journal of Motor Behavior, 23, 251-258.

Cole, K. J., \& Johansson, R. S. (1993). Friction at the digit-object interface scales the sensorimotor transformation for grip responses to pulling loads. Experimental Brain Research, 95, 523-532.

Cole, K. J., Rotella, D. L., \& Harper, J. G. (1999). Mechanisms for age-related changes of fingertip forces during precision gripping and lifting in adults. Journal of Neuroscience, 19, 3238-3247.

Edin, B. B., West Ling, G., \& Johansson, R. S. (1992). Independent control of human fingertip forces at individual digits during precision lifting. Journal of Physiology, 450, 547-564.

Flanagan, J. R. Burstedt, M. K. O., \& Johansson, R. S. (1999). Control of fingertip forces in multidigit manipulation. Journal of Neurophysiology, 81, 1706-1717.

Flanagan, J. R., Tresilian, J. R, \& Wing, A. M. (1995). Coupling of grip force and load force during arm movements with grasped objects. Neuroscience Letters, 152, 53-56.
Flanagan, J. R., \& Wing, A. M. (1997). Effects of surface texture and grip force on the discrimination of hand-held loads. Perception \& Psychophysics, 59, 111-118.

Gordon, A. M., Westling, G., Cole, K. J., \& Johansson, R. S. (1993). Memory representations underlying motor commands used during manipulation of common and novel objects. Journal of Neurophysiology, 69, 1789-1796.

HÄGER-Ross, C., Cole, K. J., \& Johansson, R. S. (1996). Grip-force responses to unanticipated object loading: Load direction reveals bodyand gravity-referenced intrinsic task variables. Experimental Brain Research, 110, 142-150.

Iто, M. (1994). Neurophysiological aspects of the cerebellar motor control system. International Journal of Neurology, 7, 162-176.

Jenmalm, P., \& Johansson, R. S. (1997). Visual and somatosensory information about object shape control manipulative fingertip forces. Journal of Neuroscience, 17, 4486-4499.

JohANSSON, R. S., \& WeSTLING, G. (1984). Roles of glabrous skin receptors and sensorimotor memory in automatic control of precision grip when lifting rougher or more slippery objects. Experimental Brain Research, 56, 550-564.

Johansson, R. S., \& WeST Ling, G. (1987). Signals in tactile afferents from the fingers eliciting adaptive motor responses during precision grip. Experimental Brain Research, 66, 141-154.

Johansson, R. S., \& Westling, G. (1988). Programmed and triggered actions to rapid load changes during precision grip. Experimental Brain Research, 71, 72-86.

Jones, L. A., \& Hunter, I. W. (1992). Changes in pinch force with bidirectional load forces. Journal of Motor Behavior, 24, 157-164.

Kumar, S., \& SimmondS, M. (1994). The accuracy of magnitude production of submaximal precision and power grips and gross motor efforts. Ergonomics, 37, 1346-1353.

TURRELL, Y. N., LI, F.-X., \& WING, A. M. (1999). Grip force dynamics in the approach to a collision. Experimental Brain Research, 128, 86-91.

Westling, G., \& Johansson, R. S. (1984). Factors influencing the force control during precision grip. Experimental Brain Research, 53, 277-284.

Wing, A. M., \& Lederman, S. J. (1998). Anticipating load torques produced by voluntary movements. Journal of Experimental Psychology: Human Perception \& Performance, 24, 1571-1581.

\section{NOTES}

1. Wood surfaces were sanded before each subject to keep the surface as consistent as possible between subjects.

2. Friction is classically said to be independent of surface area (Bowden \& Tabor, 1982).

(Manuscript received March 7, 2000; revision accepted for publication September 9, 2000.) 